\title{
Stabilité de deux chambres d'équilibre respectivement solidaires des canaux d'amenée et de fuite*
}

\section{Stability of two surge-tanks built respectively on the head-race and tail-race tunnels}

\section{ESOANDE}

CORRESPONDANT DE L'INSTTUT INGÉNIEUR I.R.T.

DIRECTEUR DE L'E.N.S.E.H.T.
PAR

E'T

\author{
R. HURON \\ MATRE DE CONFERECES A LA FACULT WES SCIENCES \\ PROFLSSEUR A L'E.N.S.E.H.T.
}

\begin{abstract}
Stabilité de réglage d'un groupe hydroélectrique encadré, à l'amont par une cheminée d'équilibre disposée à l'aval de la galerie d'amenée, à l'aval par une cheminée d'équilibre disposée à l'extrémité amont du canal de faite en charge. - Mise en équation du problème et énoncé des conditions de stabilité, suivant la méthode classique. - Parallèle entre ces conditions et les condilions de Troma relatives it chacune des deua cheminées considérées isolément. -- Applicalion: dans une installation donnée, on considere successivement cinq jeux de perte de charge dans les ounrages d'amenée et de fuite, el on étudie, dans chaque cas, la hauteut de chate limite an-dessons de laquelle l'installation est instable; compartison de celle hanteur limile avec celles résaltant de la condition de Thoma apliquée à chaque cheminée.
\end{abstract}

\begin{abstract}
Governing stabilily of a hydro-electric unit having an upstream surge-lank at the lower end of its head-race tunnel and, on the downstream side, another surge-tank at the upper end of its full-flowing tail-race tunnel. Equating of problem, and statement of stability conditions according to classical method. A parallel is drawn between these conditions and the Thoma conditions applied to each of the two surge-tanks atken separately. Application: five sets of head losses occurring in the head-race and tail-race installations are considered for a given installation and, in each case, the limit head is stadied above which the installation becomes unslable; comparison between this heal limit and these resulting from the application of the Thoma condition to each surge-tank.
\end{abstract}

\section{INTRODUCTION}

La plupart des centrales modernes comportent un canal d'amenée fonctionnant en charge, et, par suite, une chambre d'équilibre placée en tête des conduites. Certaines sont établies en souterrain, et leur canal de fuite peut, dans certaines conditions, se mettre en charge : on prévoit généralement, dans ce cas, une chambre d'équilibre à la jonction de la sortie des turbines avec le canal de fuite.

Dans la présente étude, nous examinerons les conditions de stabilité du réglage d'une turbine fonctionnant ainsi entre deux: chambres d'équilibre placées, l'une à l'extrémité aval du canal d'amenéc, l'autre à l'origine amont du canal de

(") Celte étude a fait l'objet d'unc communication à la Sociéte Hydrotechnique de France, en juin 1953. 
fuite (fig. 1). Nous supposerons ces chambres à section constante et du type ordinaire, sans itranglement.

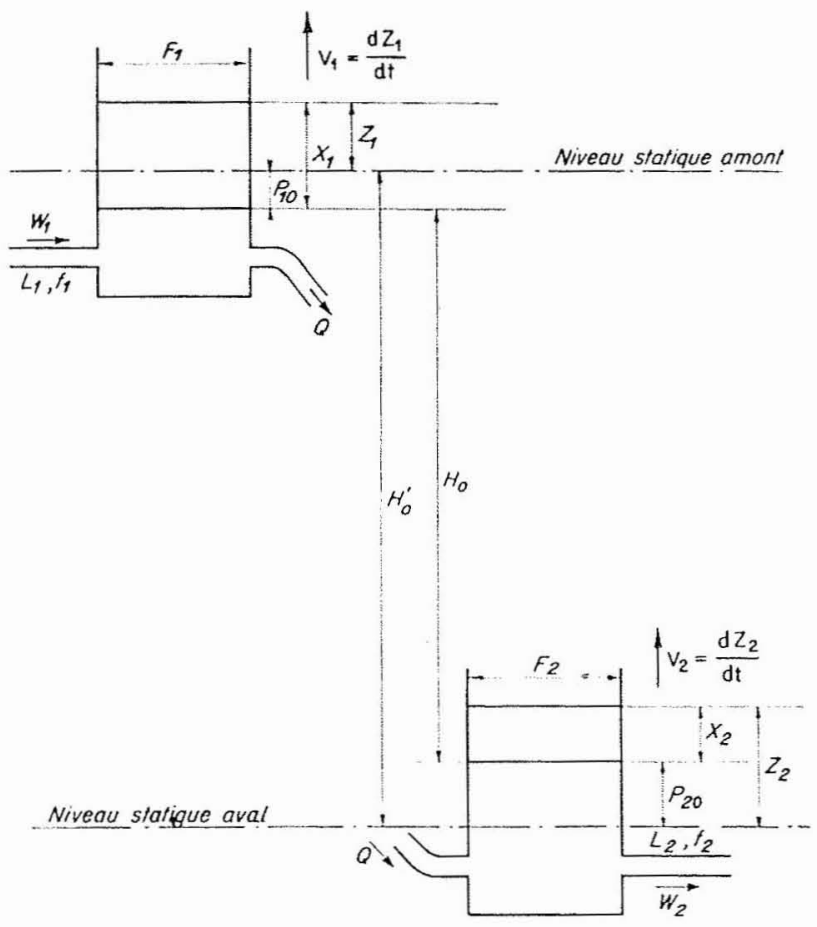

FIG. 1

C H A P I T R E 1

\section{MISE EN EQUATIONS DU PROBLÈME}

\section{NotTATIONS :}

$\mathrm{H}_{0}^{\prime}$ est la différence des cotes des niveaux statiques amont et aval.

$P_{10}, P_{20}$, les pertes de charge dans les canaux d'amenée $\left(\mathrm{P}_{16}\right)$ et de fuite $\left(\mathrm{P}_{20}\right)$ pour le débit du régime permanent considéré : $\mathbf{Q}_{0}$.

$\mathrm{H}_{0}$ la différence des cotes des plans d'eau dans les chambres d'équilibre amont et aval, lorsque le régime permanent, de débit $Q_{0}$, est établi.

$Z_{1}, X_{1}$, les cotes du plan d'eau amont, à l'instant $t$, au-dessus du niveau statique $\left(Z_{1}\right)$ ou du niveau dynamique de régime permanent $\left(\mathrm{X}_{1}=\mathrm{Z}_{\mathrm{t}}+\mathrm{P}_{10}\right)$ correspondant.

$Z_{2}, X_{2}$, les cotes du plan d'eau aval, à l'instant $t$, au-dessus du niveau statique $\left(Z_{2}\right)$ ou du niveau dynamique de régime permanent $\left(\mathrm{X}_{2}=Z_{2}-\mathrm{P}_{20}\right)$ considéré.

\section{Position du problème}

Lors d'une variation de régime, telle que celle qui aboutit au régime de débit permanent $Q_{0}$, des oscillations prennent naissance dans les deux chambres d'équilibre. Par suite, la chute sous laquelle fonctionnent les turbines, à l'instant $t$, n'est pas $\mathrm{H}_{0}$, mais :

$$
\mathrm{H}_{0}+\mathrm{X}_{1}-\mathrm{X}_{2}
$$

Avec un réglage normal, le régulateur intervient et modifie la valeur du débit, de manière à maintenir constante la puissance hydraulique, si, comme nous le supposons, la puissance électrique demeure elle-même invariable.

En négligeant les variations de rendement de la conduite et de la turbine avec le débit, on peut définir le débit $Q$ résultant de l'action du régulateur par la relation :

$$
\mathrm{Q}_{0} \mathrm{H}_{0}=\mathrm{Q}\left(\mathrm{H}_{0}+\mathrm{X}_{1}-\mathrm{X}_{2}\right)
$$

Le régulateur modifiant ainsi le débit en fonction des oscillations et ces variations de débit réagissant à leur tour sur les oscillations, le problème consiste à chercher dans quelles conditions le système sera stable, les oscillations de niveau et de débit s'amortissant plus ou moins rapidement.

\section{Introduction des grandeurs relatives}

On suppose un arrêt instantané et total des turbines et on suppose aussi que les pertes de charge n'interviennent pas. Les systèmes d'amenée et de fuite peuvent alor's être considérés indépendamment l'un de l'autre. Des oscillations se produisent dans les ehambres $\mathrm{C}_{1}$ et $\mathrm{C}_{2}$. Leurs amplitudes et périodes respectives sont désignées par :

$$
Z_{1 *}, \quad Z_{2 * *}, \quad \mathrm{~T}_{1}, \quad \mathrm{~T}_{2}
$$

Nous poserons, Q étant le débit à l'instant $t$ :

$$
\begin{aligned}
& \mathrm{U}_{10}=\mathrm{V}_{10}=\frac{\mathrm{Q}_{0}}{\mathrm{~F}_{1}} ; \quad \mathrm{W}_{10}=\frac{\mathrm{Q}_{1}}{f_{1}} ; \\
& \mathrm{U}_{1}=\frac{\mathrm{Q}}{\mathrm{F}_{1}} ; \mathrm{W}_{1}=\frac{\mathrm{Q}}{f_{1}} ; \mathrm{V}_{1}=\frac{d \mathrm{Z}_{1}}{d t} \\
& \mathrm{U}_{20}=\mathrm{V}_{20}=\frac{\mathrm{Q}_{0}}{\mathrm{~F}_{2}} ; \quad \mathrm{W}_{20}=\frac{\mathrm{Q}_{0}}{f_{2}} ; \\
& \mathrm{U}_{2}=\frac{\mathrm{Q}}{\mathrm{F}_{2}} ; \quad \mathrm{W}_{2}=\frac{\mathrm{Q}}{f_{2}} ; \mathrm{V}_{2}=\frac{d Z_{2}}{d t}
\end{aligned}
$$


On sait que l'on a :

$$
\begin{aligned}
& \mathrm{T}_{1}=2 \pi \sqrt{\frac{\mathrm{I}_{1}}{g} \frac{\mathrm{F}_{1}}{f_{1}}} ; \mathrm{T}_{2}=2 \pi \sqrt{\frac{\mathrm{L}_{2}}{g} \frac{\mathrm{F}_{2}}{f_{2}}} \\
& Z_{1 *}=\mathrm{W}_{10} \sqrt{\frac{\mathrm{L}_{1}}{g} \frac{f_{1}}{\mathrm{~F}_{1}}}=\mathrm{U}_{10} \frac{\mathrm{T}_{1}}{2 \pi} \\
& \mathrm{Z}_{2 * *}=\mathrm{W}_{20} \sqrt{\frac{\mathrm{I}_{2}}{g} \frac{f_{2}}{\mathrm{~F}_{2}}}=\mathrm{U}_{20} \frac{\mathrm{T}_{2}}{2 \pi}
\end{aligned}
$$

Si $\mathrm{P}_{1}, \mathrm{P}_{2}, \mathrm{P}_{10}, \mathrm{P}_{20}$ désignent les pertes de charge dans le canal d'amenée et dans le canal de fuite, pour le débit $Q$ et pour le débit $Q_{0}$, on peut introduire les grandeurs relatives classigues de Calame et Gaden.

$$
\begin{gathered}
z_{1}=\frac{Z_{1}}{Z_{1 *}} ; \quad x_{1}=\frac{\mathrm{X}_{1}}{Z_{1 *}} ; \quad p_{10}=\frac{\mathrm{P}_{10}}{\mathrm{Z}_{1 *}} ; \\
p_{1}=\frac{\mathrm{P}_{1}}{\mathrm{Z}_{1 *}} ; \quad h_{10}=\frac{\mathrm{H}_{0}}{\mathrm{Z}_{1 *}} \\
z_{2}=\frac{\mathrm{Z}_{2}}{\mathrm{Z}_{2 *}} ; \quad x_{2}=\frac{\mathrm{X}_{2}}{\mathrm{Z}_{2 *}} ; \quad p_{20}=\frac{\mathrm{P}_{20}}{\mathrm{Z}_{2 *}} ; \\
p_{2}=\frac{\mathrm{P}_{2}}{Z_{2 *}} ; \quad h_{20}=\frac{\mathrm{H}_{0}}{\mathrm{Z}_{2 *}} \\
u_{1}=\frac{\mathrm{U}_{1}}{\mathrm{U}_{10}} ; \quad v_{1}=\frac{\mathrm{V}_{1}}{\mathrm{~V}_{10}} ; \quad w_{1}=\frac{\mathrm{W}_{1}}{\mathrm{~W}_{10}} ; \quad t_{1}^{\prime}=\frac{t}{\mathrm{~T}_{1}} \\
u_{2}=\frac{\mathrm{U}_{2}}{\mathrm{U}_{20}} ; \quad v_{2}=\frac{\mathrm{V}_{2}}{\mathrm{~V}_{20}} ; \quad w_{2}=\frac{\mathrm{W}_{2}}{\mathrm{~W}_{20}} ; \quad t_{2}^{\prime}=\frac{t}{\mathrm{~T}_{2}}
\end{gathered}
$$

\section{Equations du système}

La relation (1) peut s'écrire, en tenant compte du fait qu'en pratique $X_{1}$ et $X_{2}$ sont petits devant $\mathrm{H}_{0}$ :

$$
\begin{gathered}
u_{1}=\frac{\mathrm{U}_{1}}{\mathrm{U}_{10}}=\frac{\mathrm{Q}}{\mathrm{Q}_{0}}=\frac{\mathrm{H}_{0}}{\mathrm{H}_{0}+\mathrm{X}_{1}-\mathrm{X}_{2}} \\
=\frac{1}{1+\frac{\mathrm{X}_{1}}{\mathrm{H}_{0}}-\frac{\mathrm{X}_{2}}{\mathrm{H}_{0}}} \# 1-\frac{\mathrm{X}_{1}}{\mathrm{H}_{0}}+\frac{\mathrm{X}_{2}}{\mathrm{H}_{0}} \\
u_{1} \# 1-\frac{\frac{\mathrm{X}_{1}}{\mathrm{Z}_{1 *}}}{\frac{\mathrm{H}_{0}}{\mathrm{Z}_{1 *}}+\frac{\mathrm{Z}_{2 *} \mathrm{X}_{2}}{\mathrm{Z}_{1 *} \mathrm{Z}_{2 *}}} \# 1-\frac{x_{1}}{\mathrm{H}_{0}}+m \frac{x_{2}}{\mathrm{Z}_{10}}(2) \\
m=\frac{\mathrm{Z}_{2 *}}{\mathrm{Z}_{1 *}}=\sqrt{\frac{\mathrm{I}_{2} f_{1} \mathrm{~F}_{1}}{\mathrm{~L}_{1} f_{2} \mathrm{~F}_{2}}}
\end{gathered}
$$

$m$ est une constante dépendant uniquement des dimensions des ouvrages et non du débit de régime $Q_{0}$ considéré.
D'une manière analogue, on aura pour $u_{2}$ :

$$
u_{2}=\frac{\mathrm{U}_{2}}{\mathrm{U}_{20}}=\frac{\mathrm{Q}}{\mathrm{Q}_{0}} \# 1-\frac{1}{m} \frac{x_{1}}{h_{20}}+\frac{x_{2}}{h_{20}}
$$

On a done : $u_{1}=u_{2}$, soit :

$$
u_{1}=u_{2}=1-\frac{x_{1}}{h_{10}}+m \frac{x_{2}}{h_{10}}=1-\frac{1}{m} \frac{x_{1}}{h_{20}}+\frac{x_{2}}{h_{20}}
$$

D’autre part, les équations classiques des oscillations dans les chambres d'équilibre donnent:

$\operatorname{Pour}\left(\mathrm{C}_{1}\right)$ :

$\left\{\begin{array}{l}v_{1} \frac{d v_{1}}{d z_{1}}+\frac{1}{2 \pi} \frac{d u_{1}}{d t_{1}}+z_{1}+p_{1}=0 \\ w_{1}=v_{1}+u_{1} ; p_{1}=p_{10} w_{1}^{2} ; z_{1}=x_{1}-p_{10}\end{array}\right.$

Pour $\left(\mathrm{C}_{2}\right)$ :

$$
\left\{\begin{array}{l}
v_{2} \frac{d v_{2}}{d z_{2}}-\frac{1}{2 \pi} \frac{d u_{2}}{d t_{2}^{\prime}}+z_{2}-p_{2}=0 \\
w_{2}=u_{2}-v_{2} ; p_{2}=p_{20} w_{2}^{2} ; z_{2}=x_{2}+p_{20}
\end{array}\right.
$$

Les relations (4), (5) et $\left(5^{\prime}\right)$ constituent les équations du système.

\section{Transformations du système}

On a : $d z_{1}=d x_{1} ; \quad d z_{2}=d x_{2}$, et d'après $(2)$ :

$$
\begin{gathered}
\frac{d u_{1}}{d t_{1}^{\prime}}=-\frac{1}{h_{10}} \frac{d x_{1}}{d t_{1}^{\prime}}+\frac{m}{h_{10}} \frac{d x_{2}}{d t_{2}^{\prime}}=-\frac{1}{h_{10}} \frac{d x_{1}}{d t_{1}^{\prime}} \\
+\frac{1}{k} \frac{1}{h_{10}} \frac{d x_{2}}{d t_{2}^{\prime}}
\end{gathered}
$$

en posant $k=\frac{\mathbf{F}_{2}}{\mathbf{F}_{1}}$ et en remarquant que :

$$
d t^{\prime}{ }_{1}=\frac{d t}{\mathrm{~T}_{1}}=\frac{\mathrm{T}_{2}}{\mathrm{~T}_{1}} \frac{d t}{\mathrm{~T}_{2}}=\frac{\mathrm{T}_{2}}{\mathrm{~T}_{1}} d t_{2}^{\prime}=k m d t^{\prime}
$$

D'autre part :

$$
\begin{gathered}
v_{1}=\frac{V_{1}}{\mathrm{U}_{10}}=\frac{\mathrm{V}_{1}}{2 \pi} \frac{\mathrm{T}_{1}}{\mathrm{Z}_{1 *}}=\frac{1}{2 \pi} \frac{d \mathrm{Z}_{1}}{d t} \cdot \frac{\mathrm{T}_{1}}{\mathrm{Z}_{1 *}} \\
=\frac{1}{2 \pi} \frac{d z_{1}}{d l^{\prime}}=\frac{1}{2 \pi} \frac{d x_{1}}{d t_{1}^{\prime}} \\
p_{1}=p_{10}\left(v_{1}+u_{1}\right)^{2}=p_{10}\left(v_{1}+1-\frac{x_{1}}{h_{10}}+m \frac{x_{2}}{h_{10}}\right)^{2} \\
p_{1}=p_{10}\left(1+\frac{1}{2 \pi} \frac{d x_{1}}{d t_{1}^{\prime}}-\frac{x_{1}}{h_{10}}+m \frac{x_{2}}{h_{10}}\right)^{2}
\end{gathered}
$$


En supposant $x_{1}, x_{2}, \frac{d x_{1}}{d t_{1}^{\prime}}$ petits, comme dans la théorie classique de Thoma, relative à une seule chambre d'équilibre placée à l'aval du canal d'amenée, on a :

$$
p_{1} \# p_{10}\left(i+\frac{1}{\pi} \frac{d x_{1}}{d t^{\prime}}-\frac{2 x_{1}}{h_{10}}+m \frac{2 x_{2}}{h_{20}}\right)
$$

Par ailleurs :

$v_{1} \frac{d v_{1}}{d z_{1}}=v_{1} \frac{d v_{1}}{d t_{1}^{\prime}} \cdot \frac{d t^{\prime}{ }_{1}}{d x_{1}}=v_{1} \frac{d v_{1}}{d t^{\prime}} \cdot \frac{1}{2 \pi v_{1}}=\frac{1}{4 \pi^{2}} \frac{d^{2} x_{1}}{d t_{1}^{\prime \prime 2}}$

En portant dans (5), on a finalement :

$$
\begin{gathered}
\frac{d^{2} x_{1}}{d t_{1}{ }^{2}}-2 \pi\left(\frac{1}{h_{10}}-2 p_{10}\right) \frac{d x_{1}}{d t^{\prime}{ }_{1}}+4 \pi^{2} \\
\left(-1-\frac{2 p_{10}}{h_{10}}\right) x_{1}=-\frac{2 \pi}{h_{10}}\left(\frac{1}{k} \frac{d x_{2}}{d t^{\prime}{ }_{2}}+4 \pi m p_{10} x_{2}\right)
\end{gathered}
$$

Par un calcul analogue, $\left(5^{\prime}\right)$ se transforme en :

$$
\begin{gathered}
\frac{d^{2} x_{2}}{d t_{2}{ }^{\prime 2}}-2 \pi\left(\frac{1}{h_{20}}-2 p_{20}\right) \frac{d x_{2}}{d t_{2}^{\prime}} \\
+4 \pi^{2}\left(1-\frac{2 p_{20}}{h_{20}}\right) x_{22}=-\frac{2 \pi}{h_{20}}\left(k \frac{d x_{1}}{d t_{1}^{\prime}}+4 \pi \frac{p_{20}}{m} x_{1}\right) \\
\text { avec : } \\
t_{1}^{\prime}=k m t_{2}^{\prime}
\end{gathered}
$$

Remaroue. - Ces équations sont, sous une forme différente, équivalentes à celles que donne M. JAEGER, dans son étude générale sur la stabilité des chambres d'équilibre, parue dans la Schweizerische Banzeitung de novembre et décembre 1943.

\section{H A P I T R E II}

\section{CONDITIONS DE STABILITÉ}

\section{Nouvelle forme des équations du problème}

Pour étudier la stabilité, il y a avantage à prendre la même variable indépendante $t$ dans les deux équations :

En remarquant que :

$$
\begin{gathered}
\frac{d x_{i}}{d t_{i}}=\frac{d x_{i}}{d t} \cdot \frac{d t}{d t_{i}}=\mathrm{T}_{i} \frac{d x_{i}}{d t} \\
\frac{d^{2} x_{i}}{d t_{i}^{2}}=\frac{d}{d t}\left(\mathrm{~T}_{i} \frac{d x_{i}}{d t}\right) \frac{d t}{d t_{i}}=\mathrm{T}_{i}^{2} \frac{d^{2} x_{i}}{d t^{2}} \quad(i=1,2)
\end{gathered}
$$

et en tenant compte que, comme on l'a vu précédemment :

$$
m k=\frac{\mathrm{T}_{2}}{\mathrm{~T}_{1}} ; \quad k=\frac{\mathrm{F}_{2}}{\mathrm{~F}_{1}}
$$

le système prend la forme:

$$
\begin{aligned}
& \frac{d^{2} x_{1}}{d t^{2}}+a_{1} \frac{d x_{1}}{d t}+b_{1} x_{1}=\mathrm{A}_{1} \frac{d x_{2}}{d t}+\mathrm{B}_{1} x_{2} \\
& \frac{d^{\prime \prime} x_{2}}{d t^{2}}+a_{2} \frac{d x_{2}}{d t}+b_{2} x_{2}=\mathrm{A}_{2} \frac{d x_{1}}{d t}+\mathrm{B}_{2} x_{1}
\end{aligned}
$$

où nous avons posé :

$$
\begin{aligned}
& a_{1}=-\frac{2 \pi}{\mathrm{T}_{1}}\left(\frac{1}{h_{10}}-2 p_{1 \mathrm{c}}\right), \quad b_{1}=\frac{4 \pi^{2}}{\mathrm{~T}_{1}{ }^{2}}\left(1-\frac{2 p_{10}}{h_{10}}\right) \\
& a_{2}=-\frac{2 \pi}{\mathrm{T}_{2}}\left(\frac{1}{h_{20}}-2 p_{20}\right), \quad b_{2}=\frac{4 \pi^{2}}{\mathrm{~T}_{2}{ }^{2}}\left(1-\frac{2 p_{20}}{h_{20}}\right) \\
& A_{1}=-\frac{2 \pi}{h_{10}} \cdot \frac{\mathrm{F}_{1}}{\mathrm{~F}_{2}} \cdot \frac{\mathrm{T}_{2}}{\mathrm{~T}_{1}{ }^{2}}, \mathrm{~B}_{1}=-8 \pi^{2} \frac{p_{10}}{h_{10}} \cdot \frac{\mathrm{F}_{1}}{\mathrm{~F}_{2}} \cdot \frac{\mathrm{T}_{2}}{\mathrm{~T}_{1}{ }^{3}} \\
& \mathrm{~A}_{2}=-\frac{2 \pi}{h_{20}} \cdot \frac{\mathrm{F}_{2}}{\mathrm{~F}_{1}} \cdot \frac{\mathrm{T}_{1}}{\mathrm{~T}_{2}{ }^{2}}, \mathrm{~B}_{2}=-8 \pi^{2} \frac{p_{20}}{h_{20}} \cdot \frac{\mathrm{F}_{2}}{\mathrm{~F}_{1}} \cdot \frac{\mathrm{T}_{1}}{\mathrm{~T}_{2}{ }^{3}}
\end{aligned}
$$

\section{Conditions nécessaires et suffisantes de stabilité}

Posons :

$$
\begin{aligned}
& \mathrm{X}_{1}(p)=\int_{0}^{+\infty} e^{-p t} x_{1}(t) d t \\
& \mathrm{X}_{2}(p)=\int_{0}^{+\infty} e^{-p t} x_{2}(t) d t
\end{aligned}
$$

Les fonctions $\mathrm{X}_{1}$ et $\mathrm{X}_{2}$ sont les transformées de Laplace des fonctions inconnues $x_{1}(t)$ et $x_{2}(t)$. D'après les formules classiques du calcul opérationnel, l'image du système (7) est:

$$
\begin{array}{r}
\mathrm{X}_{1}\left(p^{2}+a_{1} p+b_{1}\right)-\mathrm{X}_{2}\left(\mathrm{~A}_{1} p+\mathrm{B}_{1}\right) \\
=p \cdot x_{1}(0)+x_{1}^{\prime}(0)-a_{1} x_{1}(0)-\mathrm{A}_{1} x_{2}(0) \\
\mathrm{X}_{2}\left(p^{2}+a_{2} p+b_{2}\right)-\mathrm{X}_{1}\left(\mathrm{~A}_{2} p+\mathrm{B}_{2}\right) \\
=p \cdot x_{2}(0)+x_{2}^{\prime}(0)-a_{2} x_{2}(0)-\mathrm{A}_{2} x_{1}(0)
\end{array}
$$

On sait que l'étude de la stabilité repose alors sur l'étude des racines de l'équation :

$$
\Delta(p)=\left|\begin{array}{ll}
p^{2}+a_{1} p+b_{1} & -\left(\mathrm{A}_{1} p+\mathrm{B}_{1}\right) \\
-\left(\mathrm{A}_{2} p+\mathrm{B}_{2}\right) & p^{2}+a_{2} p+b_{2}
\end{array}\right|=0
$$


racines qui doivent avoir leurs parties réelles toutes négatives.

En développant le déterminant de (10), on obtient l'équation du quatrième degré :

$$
p^{4}+\alpha_{1} p^{3}+\alpha_{2} p^{2}+\alpha_{33} p+\alpha_{4}=0
$$

avee :

$$
\begin{aligned}
& \left.x_{1}=-2 \pi\left[\frac{1}{\mathrm{~T}_{1}} \frac{1}{h_{10}}-2 p_{10}\right)+\frac{1}{\mathrm{~T}_{2}}\left(\frac{1}{h_{20}}-2 p_{20}\right)\right] \\
& \alpha_{2 .}=4 \pi^{2} \frac{1}{T_{1^{2}}}\left(1-\frac{2 p_{10}}{h_{10}}\right)+\frac{1}{\mathrm{~T}_{2^{2}}}\left(1-\frac{2 p_{20}}{h_{20}}\right) \\
& +\frac{2}{\mathrm{~T}_{1} \mathrm{~T}_{2}}\left[2 p_{10} p_{20}-\left(\frac{p_{10}}{h_{20}}+\frac{p_{20}}{h_{10}}\right)\right] \text { ? } \\
& \alpha_{33}=-\frac{8 \pi^{3}}{\mathrm{~T}_{1} \mathrm{~T}_{2}}\left[\frac{1}{\mathrm{~T}_{1}}\left(\frac{1}{h_{20}}-2 p_{2,3}+\frac{4 p_{10} p_{30}}{h_{10}}\right) \mid\right. \\
& \left.+\frac{1}{\mathrm{~T}_{2}}\left(\frac{1}{h_{10}}-2 p_{10}+\frac{4 p_{10} p_{20}}{h_{20}}\right)\right] \\
& \varkappa_{-1}=\frac{16 \pi^{4}}{\mathrm{~T}_{1}{ }^{2} \mathrm{~T}_{2}^{2}}\left[1-2\left(\frac{p_{10}}{h_{10}}+\frac{p_{20}}{h_{20}}\right)\right]
\end{aligned}
$$

Les conditions nécessaires et suffisantes, pour que (11) ait ses racines à parties réelles négatives, e'est-à-dire pour que le système physique étudié soit stable sont alors (conditions de ROUTH) :

$$
\begin{gathered}
\alpha_{1}>0, \quad \alpha_{2}>0, \quad \alpha_{3}>0, \quad \alpha_{4}>0 \\
\Gamma=\alpha_{3}{ }^{*-1}-\alpha_{1}\left(\alpha_{2} \alpha_{3}-\alpha_{1} \alpha_{4}\right)<0
\end{gathered}
$$

Le probleme comportant un grand nombre de paramètres, l'étude générale des conditions (13) à partir des valeur's (12) est très compliquée, mais le passage aux valeurs numériques permet de conclure dans tous les cas.

Remaroue, - Dans le cas où il paraîtrait intéressant de garder les caractéristiques absolues du système physique, nous donnons ci-dessous, et sans expliciter les calculs, les valeurs des coefficients $\alpha_{i}$ :

$$
\begin{aligned}
\alpha_{1}= & \frac{2 g}{\mathrm{Q}_{0}}\left[\mathrm{P}_{10} \frac{f_{1}}{\mathrm{~L}_{1}}+\mathrm{P}_{20} \frac{f_{2}}{\mathrm{~L}_{2}}\right]-\frac{\mathrm{Q}_{01}}{\mathrm{H}_{0}}\left(\frac{1}{\mathrm{~F}_{1}}+\frac{1}{\mathrm{~F}_{2}}\right) \\
\alpha_{2}= & 4 g^{2} \frac{\mathrm{P}_{10} \mathrm{P}_{20}}{\mathrm{Q}^{2}} \cdot \frac{f_{1}}{\mathrm{~L}_{1}} \cdot \frac{f_{2}}{\mathrm{~L}_{2}}-\frac{2 g}{\mathrm{H}_{0}}\left(\frac{1}{\mathrm{~F}_{1}}+\frac{1}{\mathrm{~F}_{2}}\right) \\
& \left(\mathrm{P}_{10} \frac{f_{1}}{\mathrm{~L}_{1}}+\mathrm{P}_{20} \frac{f_{2}}{\mathrm{~L}_{2}}\right)+g\left(\frac{f_{1}}{\mathrm{~L}_{1} \mathrm{~F}_{1}}+\frac{f_{2}}{\mathrm{~L}_{2} \mathrm{~F}_{2}}\right) \\
\alpha_{3}= & \frac{2 g^{2}}{\mathrm{Q}_{0}} \cdot \frac{f_{1}}{\mathrm{~L}_{1}} \cdot \frac{f_{2}}{\mathrm{~L}_{2}}\left[\frac{\mathrm{P}_{10}}{\mathrm{~F}_{2}}+\frac{\mathrm{P}_{20}}{\mathrm{~F}_{1}}\right]-\frac{g \mathrm{Q}_{0}}{\mathrm{~F}_{1} \mathrm{~F}_{2} \mathrm{H}_{0}} \\
& \left.\frac{f_{2}}{\mathrm{~L}_{2}}+\frac{f_{1}}{\mathrm{~L}_{1}}\right]-\frac{4 g^{2} \mathrm{P}_{10} \mathrm{P}_{20}}{\mathrm{H}_{0} \mathrm{Q}_{0}} \frac{f_{1}}{\mathrm{~L}_{1}} \cdot \frac{f_{2}}{\mathrm{~L}_{2}}\left(\frac{1}{\mathrm{~F}_{1}}+\frac{1}{\mathrm{~F}_{2}}\right) \\
\alpha_{4}= & \frac{g^{2} f_{1} f_{2}}{\mathrm{~F}_{1} \bar{F}_{2} \mathrm{~L}_{1} \mathrm{~L}_{2}}\left(1-\frac{2 \mathrm{P}_{10}}{\mathrm{H}_{0}}-\frac{2 \mathrm{P}_{20}}{\mathrm{H}_{0}}\right)
\end{aligned}
$$

\section{Première remaroue}

La condition $x_{4}>0$ entraine la condition nécessaire :

$$
\frac{p_{10}}{h_{10}}+\frac{p_{20}}{h_{20}}<\frac{1}{2}
$$

qui est largement réalisée en pratique.

On sait que, dans le cas d'une seule chambre d'équilibre, la condition de Troma fixe une limite inférieure pour la section horizontale de la chambre.

Dans notre cas, on obtient une condition analogue en écrivant $\alpha_{1}>0$. A partir des formules (14), on obtient:

$$
\frac{1}{\mathrm{~F}_{1}}+\frac{1}{\mathrm{~F}_{2}}<\frac{2 g \mathrm{H}_{0}}{\mathrm{Q}_{0}^{2}}\left[\mathrm{P}_{10} \frac{f_{1}}{\mathrm{~L}_{1}}+\mathrm{P}_{20} \frac{f_{2}}{\mathrm{~L}_{22}}\right]
$$

Si l'on remarque que les sections limites de Thoma, relatives aux systèmes amont et aval, considérés indépendamment l'un de l'autre, sont données respectivement par les expressions :

$$
\begin{aligned}
& \mathrm{F}_{10}=\frac{1}{2 g}\left(\frac{\mathrm{Q}_{11}}{f_{1}}\right)^{2} \frac{\mathrm{L}_{1} \mathrm{f}_{1}}{\mathrm{H}_{0} \mathrm{P}_{10}} \\
& \mathrm{~F}_{20}=\frac{1}{2 g}\left(\frac{Q_{0}}{f_{2}}\right)^{2} \frac{\mathrm{L}_{2} I_{2}}{\mathrm{H}_{6} \mathrm{P}_{20}}
\end{aligned}
$$

on voit que la condition (16) peut encore s'écrire :

$$
\frac{1}{F_{1}}+\frac{1}{F_{2}}<\frac{1}{F_{10}}+\frac{1}{F_{20}}
$$

Si donc les deux systèmes satisfont à la condition de Thoma qui leur est propre :

$$
\left(\mathrm{F}_{1}>\mathrm{F}_{10}, \quad \mathrm{~F}_{2}>\mathrm{F}_{20}\right),
$$

la condition nécessaire (16) est vérifiée.

Mais cette double vérification de la condition de 'Troma n'est pas suffisante pour assurer la stabilité.

En effet, si les deux chambres sont chacune à la limite de leur condition de Thoma propre, le système physique est instable $\left(^{*}\right)$. On a, en effet, alors :

$$
\frac{1}{h_{10}}-2 p_{10}=0 \quad \frac{1}{h_{20}}-2 p_{20}=0
$$

Dans ces conditions, $\alpha_{3}$ se réduit à une valeur toujours négative:

$$
\alpha_{3}=-\frac{8 \pi^{3}}{\mathrm{~T}_{1} \mathrm{~T}_{2}} \cdot 4 p_{10} p_{20}\left(\frac{1}{\mathrm{~T}_{1} h_{10}}+\frac{1}{\mathrm{~T}_{2} h_{20}}\right)
$$

(*) Cette propriété est générale ear - $\alpha_{1}$ est la somme des racines de l'équation (11). Si cette somme est nulle, on a aussi $\left(\mathbb{R}\left(\Sigma p_{i}\right)=0\right.$ et il existe forcément des racines à parties réelles positives. 
Il en résulte que les deux conditions de Thoma :

$$
\frac{1}{h_{16}}-2 p_{10} \leqslant 0 \quad \frac{1}{h_{20}}-2 p_{20} \leqslant 0
$$

ne sont pas suffisantes, mais il est néeessaire que l'une au moins des chambres satisfasse à la condition de Thом., comme le montre immédiatement la condition $\left(16^{\prime}\right)$.

\section{Deuxième remaroue.}

Dans le cas où la hauteur de chute est assez grande pour que l'influence des oscillations du plan d'eau dans les deux chambres d'équilibre devienne négligeable, le système est évidemment toujours stable.

Il est intéressant de montrer que les conditions (13) mettent en évidence ce résultat.

Supposons, en effet, que l'on ait :

$$
\frac{1}{h_{10}}=0 \quad \frac{1}{h_{20}}=0
$$

on en déduit :

$$
\begin{array}{ll}
\alpha_{1}=4 \pi\left(\frac{p_{10}}{\mathrm{~T}_{1}}+\frac{p_{20}}{\mathrm{~T}_{2}}\right) & >0 \\
\alpha_{22}=4 \pi^{2}\left(\frac{1}{\mathrm{~T}_{1}{ }^{2}}+\frac{1}{\mathrm{~T}_{2}{ }^{2}}+\frac{4 p_{10} p_{20}}{\mathrm{~T}_{1} \mathrm{~T}_{2}}\right) & >0 \\
\alpha_{2}=\frac{16 \pi^{3}}{\mathrm{~T}_{1} \mathrm{~T}_{2}}\left(\frac{p_{20}}{\mathrm{~T}_{1}}+\frac{p_{10}}{\mathrm{~T}_{2}}\right) & >0 \\
\frac{16 \pi^{4}}{\mathrm{~T}_{1}{ }^{2} \mathrm{~T}_{2}{ }^{2}} & >0
\end{array}
$$

et, après calculs :

$$
\begin{gathered}
\Gamma=\alpha_{3}{ }^{2}-\alpha_{1}\left(\alpha_{2} \alpha_{3}-\alpha_{1} \alpha_{4}\right) \\
=-\frac{256 \pi^{6}}{\mathrm{~T}_{1} \mathrm{~T}_{2}}\left\{p_{10} p_{20}\left(\frac{1}{\mathrm{~T}_{1}{ }^{2}}-\frac{1}{\mathrm{~T}_{2}{ }^{2}}\right)^{2}\right. \\
+\frac{4 p_{10} p_{20}}{\mathrm{~T}_{1} \mathrm{~T}_{2}}\left[p_{10} p_{20}\left(\frac{1}{\mathrm{~T}_{1}{ }^{2}}+\frac{1}{\mathrm{~T}_{2}{ }^{2}}\right)+\frac{p_{10}{ }^{2}+p_{20}{ }^{2}}{\mathrm{~T}_{1} \mathrm{~T}_{2}}\right] ?
\end{gathered}
$$

oll :

$$
\Gamma=\alpha_{3}^{2}-\alpha_{1}\left(\alpha_{2} \alpha_{33}-\alpha_{1} \alpha_{4}\right)<0
$$

\section{CHA PITRE III. - APPLICATION}

Pour un projet donné, on commencera par calculer les sections $F_{1}$ et $F_{2}$ à donner aux chambres d'équilibre amont et aval, sans se préoccuper du problème de stabilité, et en tenant compte seulement de la limitation de l'amplitude des oscillations à ưne valeur convenable.
On obtiendra ainsi un système déterminé de valeurs de :

$$
\begin{array}{llllll}
\mathrm{Q}_{0} & & & \\
\mathrm{~L}_{1}, & f_{1}, & \mathrm{P}_{10}, & \mathrm{~F}_{1}, & Z_{1 *}, & \mathrm{~T}_{1} \\
\mathrm{~L}_{2}, & f_{2}, & \mathrm{P}_{2,6}, & \mathrm{~F}_{2}, & Z_{2 *}, & \mathrm{~T}_{2}
\end{array}
$$

Il restera à vérifier que les cinq conditions de stabilité :

$$
\alpha_{1}>0 \quad \alpha_{2}>0 \quad \alpha_{3}>0 \quad \alpha_{4}>0 \quad \Gamma<0
$$

sont satisfaites.

Dans le cas où il n'en serait pas ainsi, il conviendrait de recommencer le caleul en partant de valeurs supérieures des sections $F_{1}$ et $F_{2}$.

$$
* *
$$

Nous avons voulu nous rendre compte, dans divers cas particuliers, des valeurs limites $H_{01}$. de la chute de régime permanent $\mathrm{H}_{0}$, au-dessous desquelles le système devient instable, et comparer ces valeurs à celles des hauteurs limites $\mathrm{H}_{\text {or }}$ et $\mathrm{H}$ or. fournies par la condition de Тнома pour le système d'amenée d'une part $\left(\mathrm{Hor}_{\mathrm{x}}\right)$, et pour le système de fuite d'autre part $\left(\mathrm{H}_{\mathrm{OL}_{2}}\right)$.

Nous sommes partis des éléments suivants, maintenus constants :

$$
\mathrm{Q}_{0}=20 \mathrm{~m}^{3} / \mathrm{s}
$$

$$
\begin{array}{ll}
\mathrm{L}_{1}=1.000 \mathrm{~m} & \mathrm{~L}_{2}=700 \mathrm{~m} \\
f_{1}=10 \mathrm{~m}^{2} & f_{2}=10 \mathrm{~m}^{2} \\
\mathrm{~F}_{1}=40,8 \mathrm{~m}^{2} & \mathrm{~F}_{2}=79,3 \mathrm{~m}^{2} \\
\mathrm{Z}_{1 *}=10 \mathrm{~m} & \mathrm{Z}_{2 *}=6 \mathrm{~m}
\end{array}
$$

et nous avons calculé $\mathrm{H}_{\mathrm{OL}}, \mathrm{H}_{\mathrm{OL}_{1}}, \mathrm{H}_{\mathrm{OL}}$ pour cincl groupes différents de valeurs des pertes de charge $\mathrm{P}_{10}, \mathrm{P}_{20}$, soit encore, en valeurs relatives $p_{10}, p_{20}:$

\begin{tabular}{|c|c|cc|c|c|}
\hline $\operatorname{Cas~} \mathrm{N}^{\circ}$ & $\mathrm{P}_{10}$ & $\mathrm{~m}$ & $\mathrm{P}_{20} \mathrm{~m}$ & $p_{10}$ & $p_{20}$ \\
\hline 1 & 1 & 0,36 & 0,10 & 0,06 \\
\cline { 1 - 2 } 2 & 0,91 & 0,36 & 0,091 & 0,06 \\
3 & 0,833 & 0,36 & 0,0833 & 0,06 \\
4 & 0,70 & 0,36 & 0,070 & 0,06 \\
5 & 0,50 & 0,36 & 0,050 & 0,06 \\
\hline
\end{tabular}

Les planches I, II, III, IV, V représentent les courbes de variation $\alpha_{1}, \alpha_{2}, \alpha_{3}, \alpha_{4}, \Gamma$ et mettent en évidence les valeurs de $\mathrm{H}_{\mathrm{OI}}$ obtenues dans chaque cas, ainsi que celles de $\mathrm{H}_{\mathrm{OL}_{1}}$ et $\mathrm{H}_{\mathrm{OL}}$ calculées au moyen de la formule de Tномa.

Le tableau ci-après donne, pour chacun des 


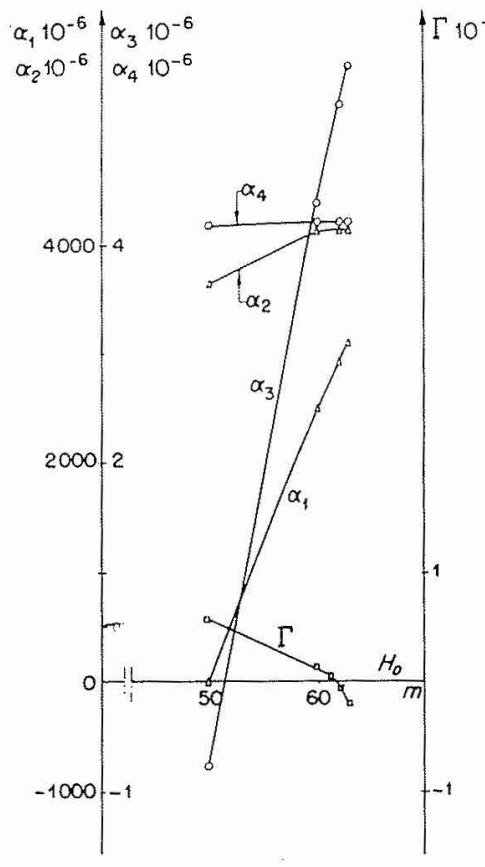

Planche I

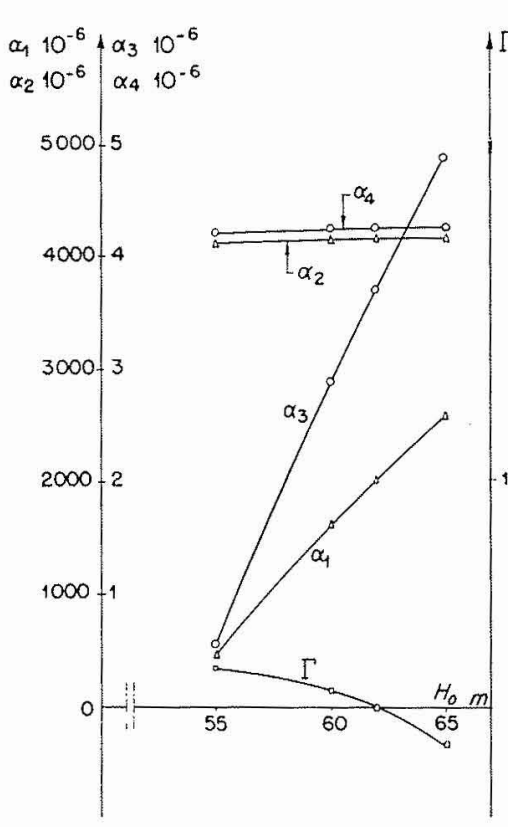

Planche II

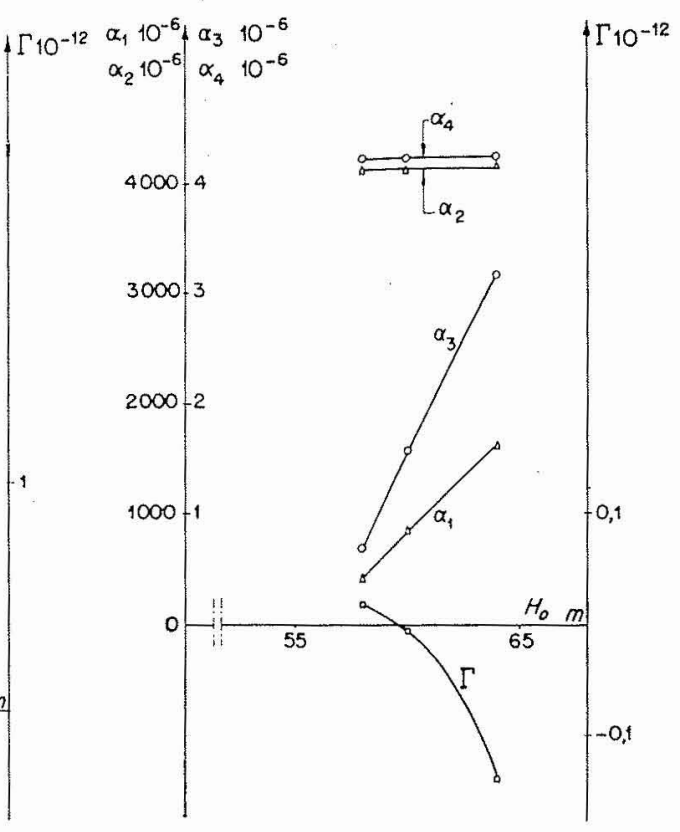

Planche III

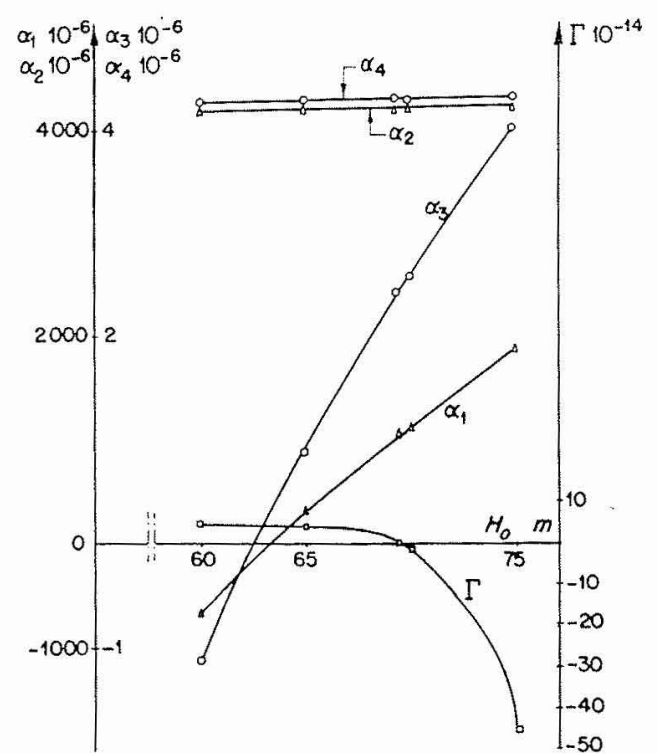

Planche IV

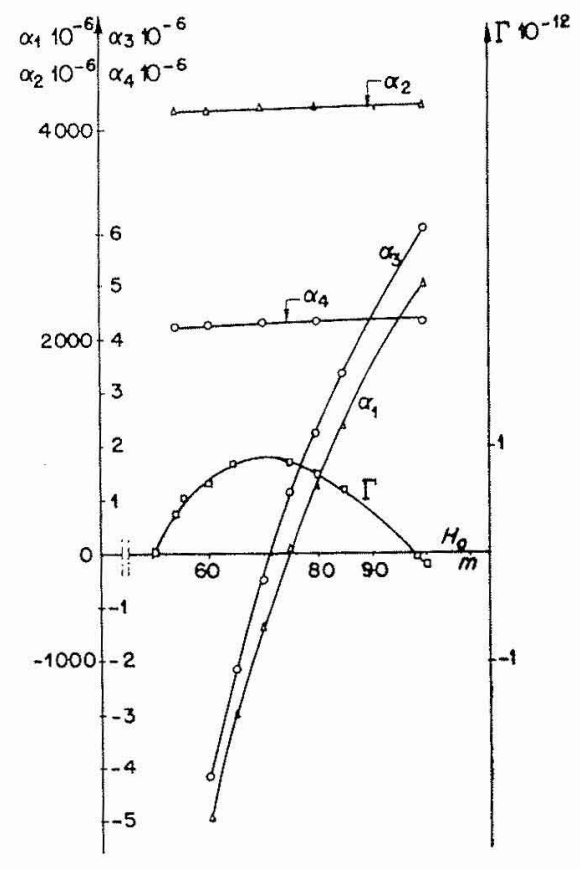

Planche V 
cinq cas étudiés, les valeurs limites $\mathrm{H}_{0 \mathrm{~L}}, \mathrm{H}_{\mathrm{OL}}$ $\mathrm{H}$ ors :

\begin{tabular}{|c|c|c|c|}
\hline Cas $N "$ & $\mathrm{H}_{\mathrm{OL}}, \quad \mathrm{m}$ & $\mathrm{HOL}_{a} \mathrm{~m}$ & $\mathrm{H}_{\mathrm{OL}} \mathrm{H}$ \\
\hline 1 & 61,8 & 50 & 50 \\
\hline 2 & 62 & 55 & 50 \\
\hline 3 & 59,7 & 60 & 50 \\
\hline 4 & 69,5 & 71,4 & 50 \\
\hline 5 & 98 & 100 & 50 \\
\hline
\end{tabular}

L'examen des résultats obtenus montre que si $\mathrm{H}_{\mathrm{H}_{2}}=\mathrm{HoL}_{2}$, leur valeur commune est inférieure à $\mathrm{H}_{\mathrm{OL}}$. Si $\mathrm{H}_{\text {ora }}$ et $\mathrm{H}_{\text {Ors }}$ sont différents, $\mathrm{H}_{\mathrm{Or}}$ est, tantôt supérieur aux deux, tantôt compris entre les deux, mais toujours supérieur au plus petit des deux. Ceci est conforme aux résultats théoriques généraux mis en évidence dans la seconde partie : si la condition de Thoma est réalisée exactement $\left(F_{1}=F_{10}, \quad F_{2}=F_{20}\right)$ pour le système d'amenée, d'une part, et pour le système de fuite, d'autre part, il n'y a pas stabilité; par ailleurs, lorsqu'il y a stabilité, la condition de Тнома est largement satisfaite, par au moins l'un des deux systèmes.

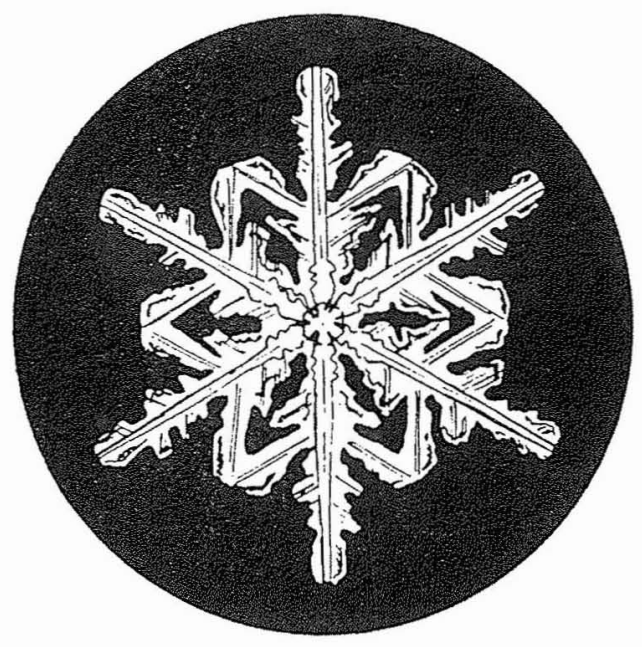

